\title{
Implementation of a molecular tumour board in LATAM: the impact on treatment decisions for patients evaluated at Instituto Alexander Fleming, Argentina
}

\author{
Martín Osvaldo Angel ${ }^{1, a}$ (D), Carmen Pupareli², Tomas Soule ${ }^{3}$, Florencia Tsou ${ }^{2, b}$ (iD, Mariano Leiva ${ }^{4}$, Federico Losco $^{1, c}$ (iD, \\ Federico Esteso ${ }^{5, \mathrm{~d}}$ (iD), Juan Manuel O 'Connor ${ }^{5, \mathrm{e}}$ (iD, Romina Luca5 ${ }^{5}$, Fernando Petracci ${ }^{6, f}$ (iD, Romina Girotti ${ }^{7}$, Yamil Damián Mahmoud $^{7, \mathrm{~g}}$ (iD, \\ Claudio Martín ${ }^{2, h}$ (iD and Matías Chacón ${ }^{8, i}$ iD
}

\begin{abstract}
${ }^{1}$ Genitourinary Oncology Unit, Instituto Alexander Fleming, Cramer 1180, Ciudad Autonoma de Buenos Aires, C1426ANZ, Argentina ${ }^{2}$ Thoracic Oncology Unit, Instituto Alexander Fleming, Cramer 1180, Ciudad Autonoma de Buenos Aires, C1426ANZ, Argentina

${ }^{3}$ Sarcoma and Melanoma Oncology Unit, Instituto Alexander Fleming, Cramer 1180, Ciudad Autonoma de Buenos Aires, C1426ANZ, Argentina

${ }^{4} \mathrm{Head}$ and Neck Oncology Unit, Instituto Alexander Fleming, Cramer 1180, Ciudad Autonoma de Buenos Aires, C1426ANZ, Argentina

${ }^{5}$ Gastrointestinal Oncology Unit, Instituto Alexander Fleming, Cramer 1180, Ciudad Autonoma de Buenos Aires, C1426ANZ, Argentina

${ }^{6}$ Breast Cancer Unit, Instituto Alexander Fleming, Cramer 1180, Ciudad Autonoma de Buenos Aires, C1426ANZ, Argentina

${ }^{7}$ Laboratorio de Inmuno-Oncología Traslacional, Instituto de Biología y Medicina Experimental (IBYME), Vuelta de Obligado 2490, Ciudad Autonoma de Buenos Aires, C1428ADN, Argentina

${ }^{8}$ Clinical Oncology, Instituto Alexander Fleming, Cramer 1180, Ciudad Autonoma de Buenos Aires, C1426ANZ, Argentina

ahttps://orcid.org/0000-0002-1463-8887
\end{abstract}

bhttps://orcid.org/0000-0002-0322-0434

chttps://orcid.org/0000-0001-5084-3012

dhttps://orcid.org/0000-0003-1977-9846

ehttps://orcid.org/0000-0002-6975-5466

fhttps://orcid.org/0000-0002-7701-3331

ghttps://orcid.org/0000-0001-7254-5892

hhttps://orcid.org/0000-0003-4135-7332

ihttps://orcid.org/0000-0001-6872-4185

\section{Abstract}

Background: The role of the molecular tumour board (MTB) is to recommend personalised therapy for patients with cancer beyond standard-of-care treatment. A comprehensive molecular analysis of the tumour in a molecular pathology laboratory is important for all targeted therapies approaches. Here we report the 1-year experience of the Instituto Alexander Fleming Molecular Tumour Board.

Patients and methods: The MTB of the Instituto Alexander Fleming was launched in December 2019 in a monthly meeting. In each interactive monthly session, five cases were presented and discussed by the members. These cases were referred by the treating oncologists. The MTB recommendations were sent to each physician individually, and to the rest of the meeting participants. This was discussed with the patients/families by the treating oncologist. The final decision to choose therapy was left to the treating physicians. Of the 32 patients presented at MTB, 28 (87.5\%) had potentially actionable alterations and only 4 (12.5\%) had no actionable mutation. Six (19\%) patients received a local regulatory agency approved drug recommendation, nine (28\%) patients received an off-label approval treatment recommendation and three (9\%) patients did not receive the treatment due to access and reimbursement of the drug.

Correspondence to: Martin Angel Email: mangel@alexanderfleming.org

ecancer 2021, 15:1312

https://doi.org/10.3332/ecancer.2021.1312

Published: 01/11/2021

Received: 03/07/2021

Publication costs for this article were supported by ecancer (UK Charity number 1176307).

Copyright: () the authors; licensee ecancermedicalscience. This is an Open Access article distributed under the terms of the Creative Commons Attribution License (http:// creativecommons.org/licenses/by/4.0), which permits unrestricted use, distribution, and reproduction in any medium, provided the original work is properly cited. 
Conclusion: In most of the cases evaluated, the MTB was able to provide treatment recommendations based on targetable genetic alterations. Molecular-guided extended personalised patient care is effective for a small but clinically significant proportion of patients in challenging clinical situations. We believe that the implementation of a MTB is feasible in the Latin America (LATAM) region.

Keywords: molecular tumour board, precision medicine, molecular profile

\section{Background}

The identification of molecular targets has allowed the personalisation of medicine. The first example arises from the discovery of the c-Kit activation pathway in gastrointestinal (GI) tumours and the use of imatinib for its treatment [1]. Only one patient was enough to demonstrate this proof of concept; this is, from our point of view, the birth of precision medicine. The use of targeted therapies in oncology is gaining ground, and oncologists are striving every day to implement them to change the paradigm of oncology practice that requires large-scale clinical trials to change therapeutic behaviours [2].

Later, with advances in the understanding of tumour biology and the discovery of oncogenic activation pathways, the oncologist began to implement biomarker-based treatment strategy. The landmark milestone of genomics in oncology was The Cancer Genome Atlas project that was able to molecularly characterise over 20,000 tumours [3]. Different tumour models have followed this molecular characterisation strategy and multiple therapeutic targets have been identified [4]. Some examples of malignant melanoma arise with the identification of activating mutations in the BRAF oncogene, being one of the most frequent genetic alterations in melanoma (50\%). BRAF mutations result in the constitutive activation of the BRAF kinase and, consequently, the subsequent activation of the mitogen-activated protein kinase signalling pathway that regulates proliferation, growth and cell differentiation $[5,6]$. Treatment with BRAF and MEK inhibitors has increased overall survival in melanoma.

Lung cancer is another good example for molecular subtyping. The epidermal growth factor receptor (EGFR) represents an important signalling pathway that regulates tumorigenesis and cell survival and is frequently overexpressed in the development and progression of non-small cell lung cancer (NSCLC) [7, 8]. This was followed by the identification of other activation pathways such as ALK, ROS, MET, BRAF and RET alterations [9-12]. Multiple targeted therapies have been approved for these molecular alterations.

With the landmark approvals of pembrolizumab for the treatment of patients whose tumours have high microsatellite instability, larotrectinib and entrectinib for those harbouring neurotrophic tyrosine receptor kinase (NTRK) fusions, a regulatory pathway has been created to facilitate the approval of histology-agnostic indications [13, 14].

The molecular similarities between histology and anatomical location of cancer types provide basis for developing strategies for future therapeutic research. This approach can only be carried out by a multidisciplinary team with a comprehensive molecular vision: the molecular tumour board (MTB). The role of the MTB is to recommend personalised therapy for patients with cancer beyond standard-of-care treatment [15]. A comprehensive molecular analysis of the tumour in a molecular pathology laboratory is important for all targeted therapies approaches. However, the interpretation of the molecular results is crucial and potential therapeutic conclusions can only be drawn by considering the clinical situation and within a setting of oncological experience. MTBs are becoming more common worldwide.

We established an MTB to interpret individual patient's tumour genetic profiles and provide treatment recommendations. Here we report the 1-year experience of the Instituto Alexander Fleming Molecular Tumour Board. We are a private oncological institute in Buenos Aires, Argentina, specialised in solid and haematological tumours with a higher volume of patients per year.

\section{Patients and methods}

The MTB of the Instituto Alexander Fleming called IAF_Molecular was launched in December 2019 in a monthly meeting. The MTB involves medical oncologists, pathologist, clinical trials investigators, genetic counsellors, molecular biologists and bioinformatics professionals. In 
each interactive monthly session, five cases were presented and discussed by the members. These cases were referred by the treating oncologists. All information was de-identified according to the local ethics committee. Patients were informed about the MTB decision-making process when their case was referred for discussion. Patient's information required: primary tumour type, stage at diagnosis, previous lines of treatment, performance status, molecular and/or immune-histochemical biomarkers, etc. This was followed by a discussion of the results of the molecular profile and the implications for each case by the molecular biologist. The information discussed included the alterations detected in each sample, their level of characterisation and potential actionability. Targeted therapies or immunotherapies that matched each detected and approved alteration in the patient's tumour type or in another tumour type were also analysed, as well as the open enrolment of clinical trials with genomic compatibility. This was only a consultative discussion. The MTB recommendations were sent to each physician individually, and to the rest of the meeting participants, by email and were kept on the shared drive for future reference. This was discussed with the patients/families by the treating oncologist. The final decision to choose therapy was left to the treating physicians (Figure 1).

Regarding the comprehensive genomic profiling (CGP) tests, all patients presented at MTB had performed a commercially available test as FoundationOne ${ }^{\circ} \mathrm{CDx}$, (Liquid or Heme) or Caris ${ }^{\circledR}$.

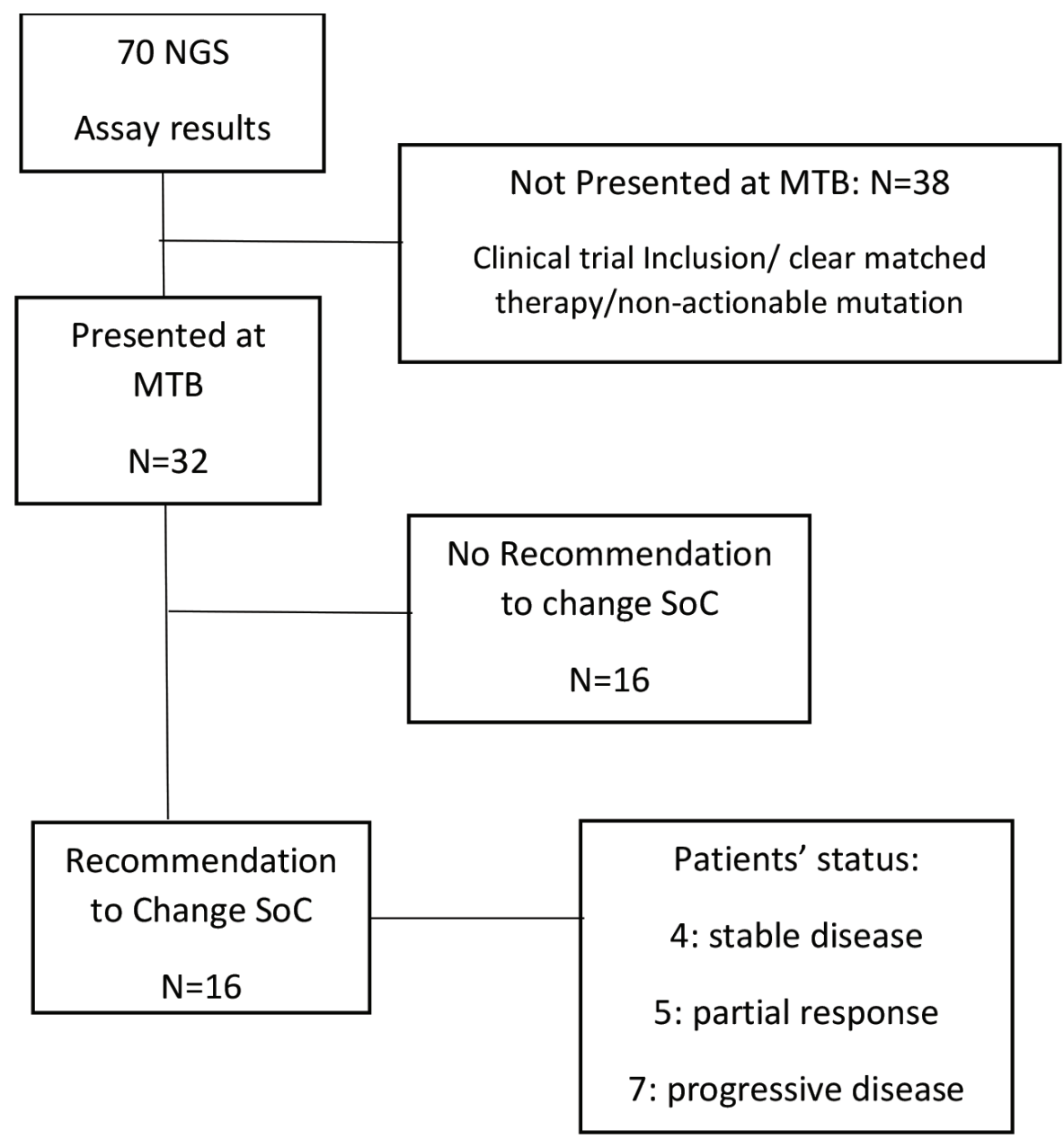

Figure 1. Workflow of the MTB at Instituto Alexander Fleming (period: December 2019 through December 2020). SoC, Standard of care; NGS, Next generation sequencing; MTB, Molecular tumour board. 


\section{Results}

\section{Patient characteristics}

Patients whose cases were selected for CGP had a range of different solid tumour types ( $n=70)$ : NSCLC $(n=13)$, breast cancer $(n=4)$, GI cancer $(n=34)$, genitourinary cancer $(n=3)$, unknown primary $(n=3)$, sarcomas $(n=3)$, head and neck cancer $(n=6)$ and gynaecological cancer $(n=4)$. Patients who were candidates for any of the open clinical trials at our site, or non-actionable mutation detected, and with clear matches to Food and Drug Administration as well as national regulatory agency (Administración Nacional de Medicamentos, Alimentos y Tecnología Médica) approved therapies in their tumour type were excluded for MTB discussion. Cases were selected for presentation to the MTB where the specific genomic mutation was not a direct match to an approved treatment or available clinical trial. Patients included for MTB discussion $(n=32)$. Amongst patients presented, the majority were NSCLC $(28 \%, 9 / 32)$ followed by breast cancer $(12.5 \%, 4 / 32)$, colorectal cancer (12.5\%, 4/32), pancreatic adenocarcinoma (12.5\%, 4/32), carcinoma of unknown primary (CUP) (9\%, 3/32) and others tumour types $(25 \%, 8 / 32)$ including oesophageal carcinoma, sarcomas, mesothelioma, cholangiocarcinoma, kidney and prostate cancer. Demographics of patients discussed are represented in Table 1. Median age was 57.8 years (interquartile range (IQR): $52-68.7$ ) and 1:1 male to female ratio.

Median number of previous treatments lines was 2 (IQR: 2-3). All patients presented an Eastern Cooperative Oncology Group (ECOG) performance score of 0 at the time of MTB presentation.

Table 1. Clinical and genomic characteristics of patients' treatment based on MTB discussion.

\begin{tabular}{|c|c|c|c|c|c|}
\hline Patient & Diagnosis & Genomic alteration & MTB recommendation & PFS (months) & Best response \\
\hline 1 & NSCLC & EGFR K745-E746ins & Afatinib & 29.25 & SD \\
\hline 2 & NSCLC & GNAS p.Q227L & Trametinib & 10.75 & PD \\
\hline 3 & CRC & ERBB2 & Trastuzumab/pertuzumab & 1.58 & PD \\
\hline 4 & NSCLC & ALK & Alectinib & 38.08 & PR \\
\hline 5 & Pancreatic adenocarcinoma & ATM & Olaparib & 9.67 & PR \\
\hline 6 & Urothelial & STK11 & Everolimus & 1.55 & PD \\
\hline 7 & CRC & BRCA1 & Olaparib & Never done & PR \\
\hline 8 & Ductal carcinoma (breast) & ERBB2 & Trastuzumab Pertuzumab & 13.58 & PD \\
\hline 9 & CUP & KIT & Avapritinib & 17.00 & PD \\
\hline 10 & NSLC & EGFR & Osimertinib & 37.75 & PR \\
\hline 11 & mCRPC & AR amplification & Abiraterone & Never done & PR \\
\hline 12 & NSCLC & ALK fusion & Lorlatinib & 23.83 & PD \\
\hline 13 & CUP & ERBB2 & Trastuzumab/pertuzumab & 15.25 & PD \\
\hline 14 & Ductal carcinoma (breast) & FGF19 amplification & Infigratinib & Never done & SD \\
\hline 15 & Bone sarcoma & FGFR1 amplification & Pazopanib & 7.42 & SD \\
\hline 16 & Cholangiocarcinoma & IDH1 & Ivosidenib & Never done & SD \\
\hline
\end{tabular}

NSCLC, Non-small cell lung carcinoma; CRC, Colorectal carcinoma; CUP, Carcinoma of unknown primary; mCRPC, Metastatic castration resistant prostate cancer; SD, Stable disease; PD, Progressive disease; PR, Partial response; PFS, Progression-free survival; AR, androgen receptor. 


\section{Genomic alterations and potential treatment options identified}

Of the 32 patients presented at MTB, 28 patients (87.5\%) had potentially actionable alterations and only 4 (12.5\%) had no actionable mutation (Figure 2). Fifteen cases (46.8\%) had alterations with therapeutic benefit in their tumour type and 17 (53.1\%) had alterations with matched therapy in another tumour type. In eight patients (25\%), more than one potentially actionable alterations were identified. Twenty cases (62.5\%) had an identified resistance alteration (Figure 3). All the patients presented at MTB had microsatellite status stable or undetermined. The median number of mutations per megabase determined as tumour mutational burden (TMB) was 4.3 mut/mb (IQR: 1.0-5.65) (Figure 4). Only two patients have high TMB.

\section{Treatment assignments and patient outcomes}

Treatment recommendations in MTB are detailed in Table 1. Six (19\%) patients received a local regulatory agency approved drug recommendation, nine (28\%) patients received an off-label approval treatment recommendation and three (9\%) patients did not receive the treatment due to access and reimbursement of the drug. One patient received an un-approved drug under an early access programme.

Amongst patients presented at the MTB, and those in which treatment recommendation was adopted, the median progression free survival was 15.25 months. According to best response obtained, median PFS in patients achieving disease control (SD + PR) was 16.4 months (95\% confidence interval: $1.1-19.4$ months, $\mathrm{p}=0.04)$.

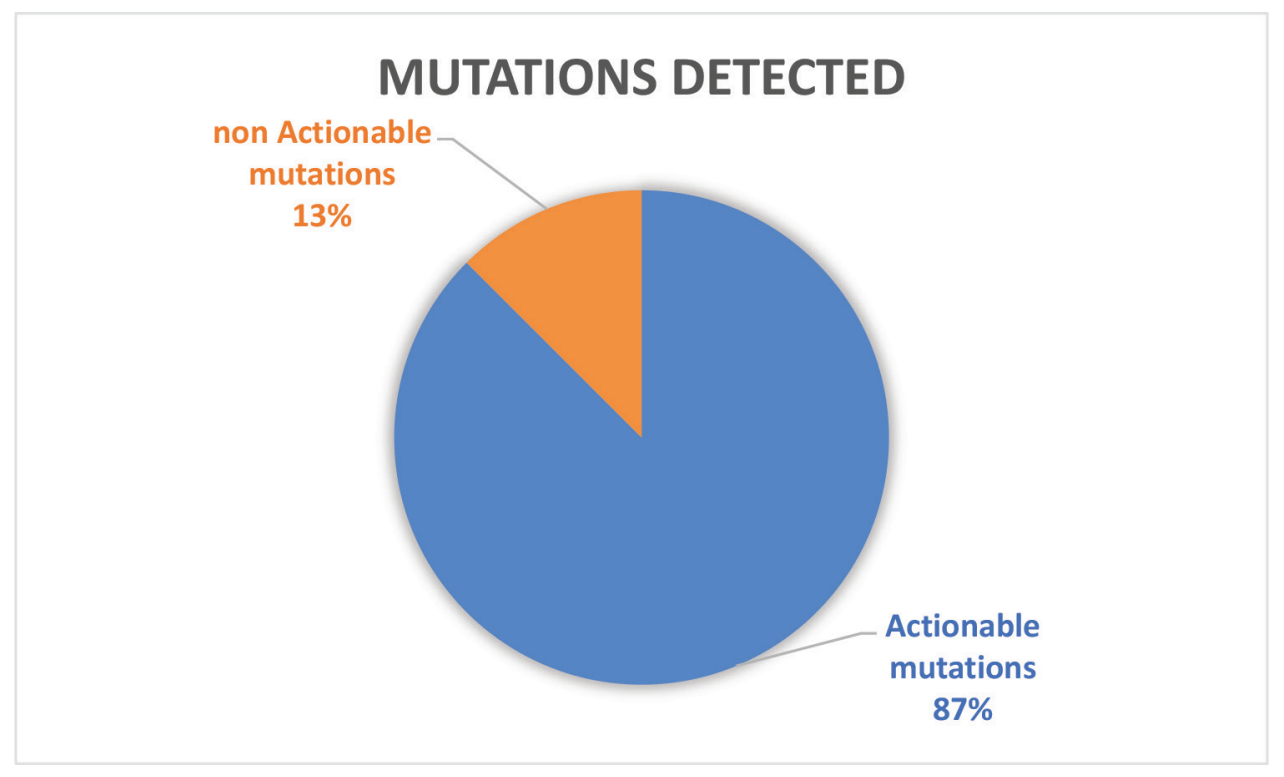

Figure 2. Number of patients with actionable mutations presented at MTB. 


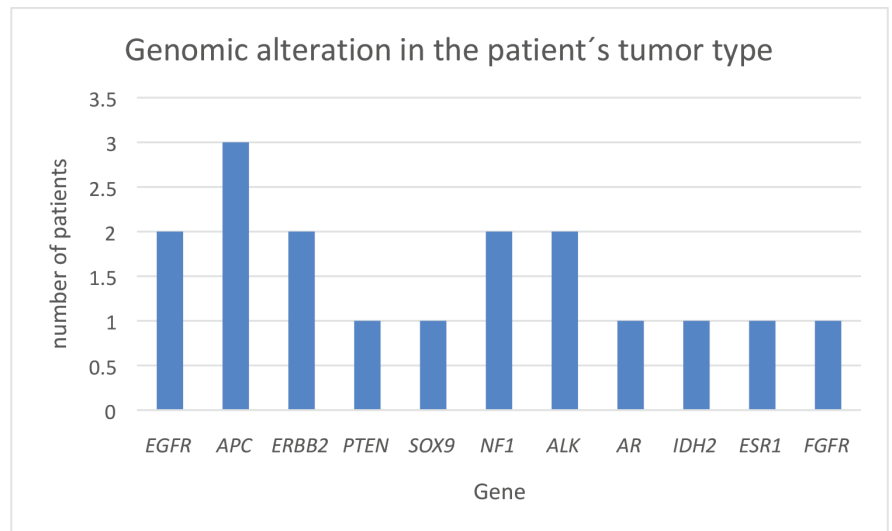

(a)

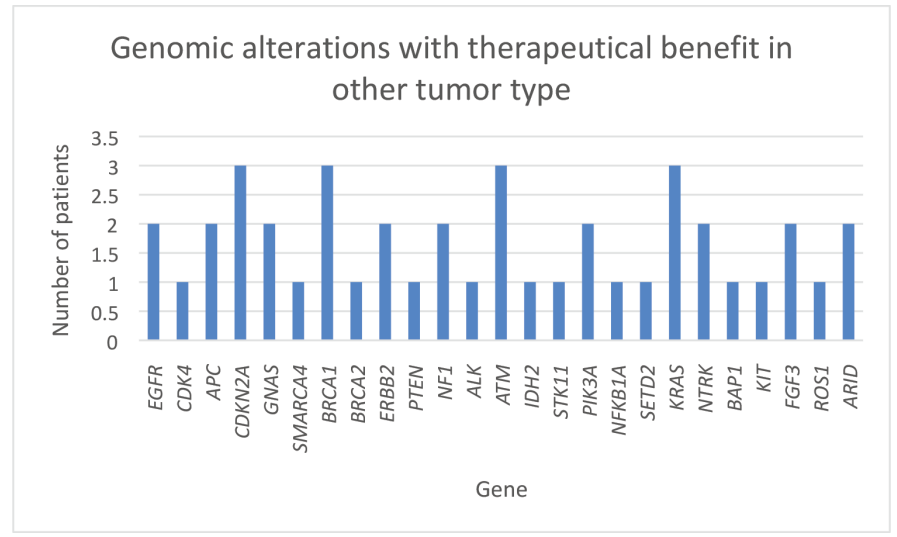

(b)

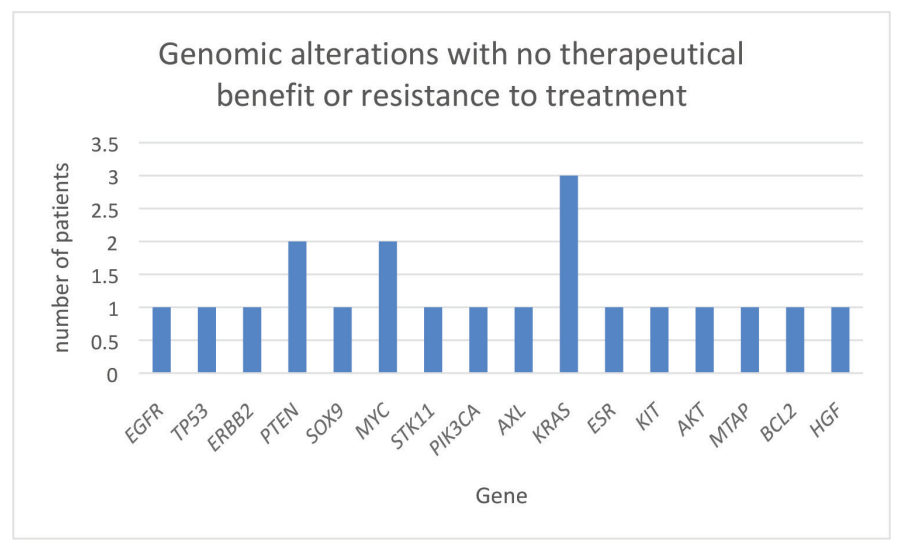

(c)

Figure 3. Genomics alterations detected in patients presented at MTB. (a): Genomic alteration with potential benefit in patient's tumour type. (b): Genomic alterations with potential therapeutical benefit in other tumour types. (c): Genomic alterations with no therapeutic benefit or resistance to treatment. 


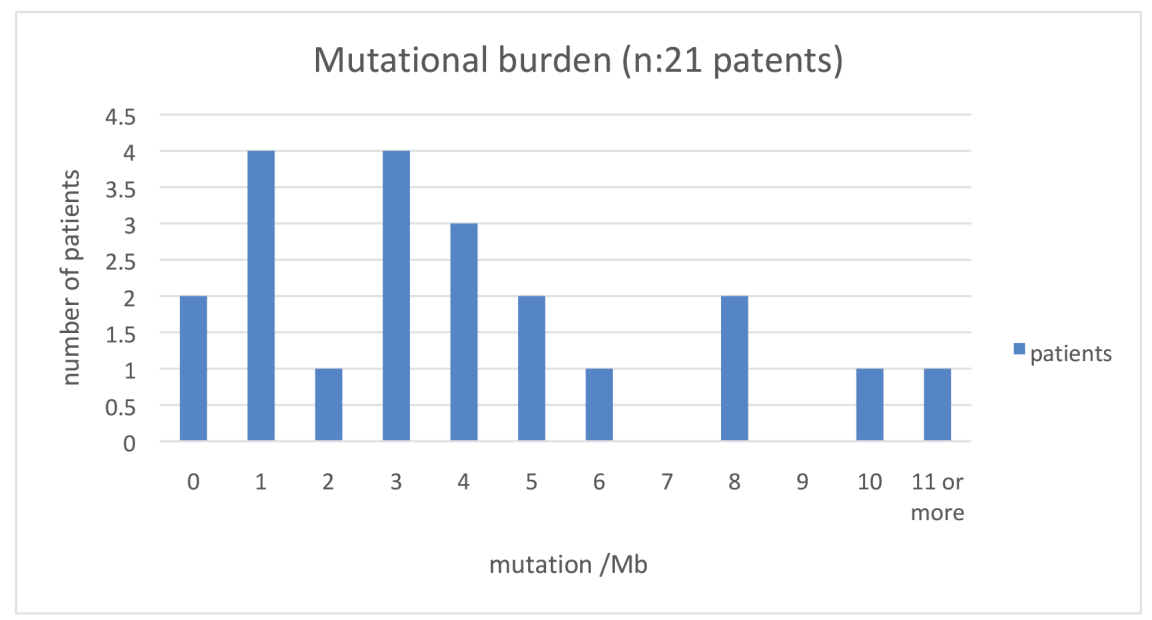

Figure 4. Number of patients according to mutations/Mb.

\section{Discussion}

We believe that the implementation of a MTB committee in our region is feasible and also necessary. The region comprises almost $8.5 \%$ of the world's population. During the last decades, Latin America (LATAM) has experienced an increase in life expectancy and the subsequent ageing of the population. This change inevitably results in a higher incidence of cancer [16, 17]. Regarding molecular profile of different tumours, although there is a clear country and continental variability in terms of frequency, this difference is not significant and the overall incidence of different targets mutations in LATAM does not differ from the rest of the world. (EGFR and ALK in NSCLC [18], BRCA1 and BRCA2 in breast and ovarian cancer [19] and homologous recombinant deficiency in prostate cancer [20].) The request for genomic profiling is increasingly frequent worldwide [21], also in our institution, mainly as a diagnostic tool, so clinical oncologists need the MTB space for a better understanding of the report and to identify the oncogenic pathway that is dominating in the patient. Amongst the advantages that can be identified by having an MTB, is to have a common space for discussion of clinical cases, and to share the scientific evidence available in a tumour model to be applied in another type of tumour with an identified mutation. This cross communication between different branches of oncology allows us to optimise treatment and guide the best therapy to our patients.

The presence of activating mutations of certain signalling pathways are the ones that dominate the progression of the disease; therefore, the acquisition of new knowledge of signalling pathways and the action of these pathways to determine the treatment as well as to identify drug resistance mutations are also useful. In this sense, the incorporation of molecular biologists and bioinformaticians to our MTB also helped us with this point. Amongst the patients presented at the MTB, at least 16 known resistance mutations were identified, so the treatment recommendation issued by the committee was to continue with standard treatment despite the availability of an actionable mutation.

Another point of interest of our MTB was the incorporation of a genetic counsellor in oncology, which helped us to identify mutations that potentially present a germline implication and require follow-up of the patient and family members [22]. Amongst those present at MTB, at least five patients were identified, who were referred for germline testing, mainly when mutations in the BRCA and ATM genes were identified.

The strongest point of our committee that we can identify as very helpful is the ability to collect individual patient data and perform an aggregate analysis according to the identified mutations. This allowed us to standardise therapeutic behaviours in certain types of tumours, as well as to be able to design strategies for the treatment of certain types of cancer. 
Differences in MTB interpretation and recommendation vary by centre, access to the clinical trial and other factors [23-25]. To date, there are no validated workflows to provide recommendations for the identified molecular targets. The strength of our committee is the ability to collect data from individual patients and perform an aggregate analysis according to the identified mutations. This allowed us to standardise therapeutic behaviours in certain tumours types, as well as to be able to design strategies for the treatment of certain types of cancer.

\section{Conclusion}

In most of the cases evaluated, MTB was able to provide treatment recommendations based on actionable genetic alterations. Although the number of patients presented in our report is still small, our observations suggest that MTBs bring together relevant expertise and are crucial to patient care.

\section{Conflicts of interest}

None of the authors have conflicts of interest to declare.

\section{Funding}

The authors have not declared a specific grant for this research from any funding agency in the public, commercial or not-for-profit sectors.

\section{References}

1. Joensuu H, Roberts PJ, and Sarlomo-Rikala M, et al (2001) Effect of the tyrosine kinase inhibitor STI571 in a patient with a metastatic gastrointestinal stromal tumor N Engl J Med 344(344) 1052-1056

2. Moscow JA, Fojo T, and Schilsky RL (2017) Clinical evidence of the safety and efficacy of treatments, demonstrated by prospective clinical trials, provides the framework for much of oncology practice Nat Rev Clin Oncol

3. Hoadley KA, Yau C, and Hinoue T, et al (2018) Cell-of-origin patterns dominate the molecular classification of 10,000 tumors from 33 types of cancer Cell 173(2) 291-304 https://doi.org/10.1016/j.cell.2018.03.022 PMID: 29625048 PMCID: 5957518

4. Toss A, and Cristofanilli M (2015) Molecular characterization and targeted therapeutic approaches in breast cancer Breast Cancer Res 17(1) 60 https://doi.org/10.1186/s13058-015-0560-9

5. Chapman PB, Robert C, and Larkin J, et al (2017) Vemurafenib in patients with BRAFV600 mutation-positive metastatic melanoma: final overall survival results of the randomized BRIM-3 study Ann Oncol 28(10) 2581-2587 https://doi.org/10.1093/annonc/mdx339 PMID: 28961848 PMCID: 5834156

6. Fraser VJ, and Powderly WG (1995) Risks of HIV infection in the health care setting Annu Rev Med 46 203-211 https://doi.org/10.1146/ annurev.med.46.1.203

7. Fukuoka M, Wu YL, and Thongprasert S, et al (2011) Biomarker analyses and final overall survival results from a phase III, randomized, open-label, first-line study of gefitinib versus carboplatin/paclitaxel in clinically selected patients with advanced non - small-cell lung cancer in Asia (IPASS) J Clin Oncol 29(21) 2866-2874 https://doi.org/10.1200/JCO.2010.33.4235

8. Sato M, Shames DS, and Gazdar AF, et al (2007) A translational view of the molecular pathogenesis of lung cancer J Thorac Oncol 2(4) 327-343 https://doi.org/10.1097/01.JTO.0000263718.69320.4c 
9. Ou SHI, Ahn JS, and De Petris L, et al (2016) Alectinib in crizotinib-refractory alk-rearranged non-small-cell lung cancer: a phase II global study J Clin Oncol 34(7) 661-668 https://doi.org/10.1200/JCO.2015.63.9443

10. Solomon BJ, Mok T, and Kim DW, et al (2014) First-line crizotinib versus chemotherapy in ALK-positive lung cancer N Engl J Med 371(23) 2167-2177 https://doi.org/10.1056/NEJMoa1408440 PMID: 25470694

11. Drilon A, Oxnard GR, and Tan DSW, et al (2020) Efficacy of selpercatinib in RET fusion-positive non-small-cell lung cancer N Engl J Med 383(9) 813-824 https://doi.org/10.1056/NEJMoa2005653 PMID: 32846060 PMCID: 7506467

12. Davies KD, Ng TL, and Estrada-Bernal A, et al (2017) Dramatic response to Crizotinib in a patient with lung cancer positive for an HLADRB1-MET gene fusion JCO Precis Oncol 2017(1) 1-6

13. Drilon A, Laetsch TW, and Kummar S, et al (2018) Efficacy of larotrectinib in TRK fusion-positive cancers in adults and children N Engl J Med 378(8) 731-739 https://doi.org/10.1056/NEJMoa1714448 PMCID: 5857389

14. Doebele RC, Drilon A, and Paz-Ares L, et al (2020) Entrectinib in patients with advanced or metastatic NTRK fusion-positive solid tumours: integrated analysis of three phase 1-2 trials Lancet Oncol 21(2) 271-282 https://doi.org/10.1016/S1470-2045(19)30691-6 PMCID: 7461630

15. Trivedi H, Acharya D, and Chamarthy U, et al (2019) Implementation and outcomes of a molecular tumor board at Herbert-Herman cancer center, sparrow hospital Acta Medica Acad 48(1) 105 https://doi.org/10.5644/ama2006-124.247

16. Bray F, Ferlay J, and Soerjomataram I, et al (2018) Global cancer statistics 2018: GLOBOCAN estimates of incidence and mortality worldwide for 36 cancers in 185 countries CA Cancer J Clin 68(6) 394-424 https://doi.org/10.3322/caac.21492 PMID: 30207593

17. Raez LE, Cardona AF, and Santos ES, et al (2018) The burden of lung cancer in Latin-America and challenges in the access to genomic profiling, immunotherapy and targeted treatments Lung Cancer 119(9) 7-13 https://doi.org/10.1016/j.lungcan.2018.02.014

18. Arrieta O, Cardona AF, and Martín C, et al (2015) Updated frequency of EGFR and KRAS mutations in non-small-cell lung cancer in Latin America: the Latin-American consortium for the investigation of lung cancer (CLICaP) J Thorac Oncol 10(5) 838-843 https://doi. org/10.1097/JTO.0000000000000481 PMID: 25634006

19. Jara L, Morales S, and de Mayo T, et al (2017) Mutations in BRCA1, BRCA2 and other breast and ovarian cancer susceptibility genes in Central and South American populations Biol Res 50(1) 35 https://doi.org/10.1186/s40659-017-0139-2

20. de Bono J, Mateo J, and Fizazi K, et al (2020) Olaparib for metastatic castration-resistant prostate cancer N Engl J Med 382(22) 20912102 https://doi.org/10.1056/NEJMoa1911440 PMID: 32343890

21. Patel NM, Michelini VV, and Snell JM, et al (2018) Enhancing next-generation sequencing-guided cancer care through cognitive computing Oncologist 23(2) 179-185 https://doi.org/10.1634/theoncologist.2017-0170 PMCID: 5813753

22. Rodríguez-Balada M, Roig B, and Melé M, et al (2020) Identification of germline pathogenic variants in DNA damage repair genes by a next-generation sequencing multigene panel in BRCAX patients Clin Biochem 76 17-23 https://doi.org/10.1016/j.clinbiochem.2019.11.014

23. Rieke DT, Lamping M, and Schuh M, et al (2018) Comparison of treatment recommendations by molecular tumor boards worldwide JCO Precis Oncol 21-14

24. Tafe LJ, Gorlov IP, and Abreu FB, et al (2015) Implementation of a molecular tumor board: the impact on treatment decisions for 35 patients evaluated at Dartmouth-Hitchcock Medical Center Oncologist 20(9) 1011-1018 https://doi.org/10.1634/theoncologist.2015-0097 PMID: 26205736 PMCID: 4571816

25. Schwaederle M, Parker BA, and Schwab RB, et al (2014) Molecular tumor board: the university of California San Diego Moores cancer center experience Oncologist 19(6) 631-636 https://doi.org/10.1634/theoncologist.2013-0405 PMID: 24797821 PMCID: 4041669 